\title{
Comparison of Online Dementia Information in Chinese and in English Languages
}

\author{
John T. Tsiang ${ }^{1}$, Benjamin K. Woo ${ }^{2}$ \\ 1. Medicine, Case Western Reserve University School of Medicine 2. UCLA
}

Corresponding author: Benjamin K. Woo, bewoo@dhs.lacounty.gov

\begin{abstract}
\section{Introduction}

There is a deficit of avenues for obtaining dementia information in the Asian American community. This study aims to compare the content and quality differences between websites providing information on dementia as found by a Google search conducted both in simplified Chinese characters and in English.
\end{abstract}

\section{Methods}

A Google search was performed for the phrase "dementia" in simplified Chinese characters and in English. The resultant websites were categorized by whether they were commercial in nature, the type of website, and whether the website provided an explanation of dementia signs and symptoms. The quality of the websites was assessed via readability and the Health on the Net Code of Conduct (HONcode). Chi-squared analyses were performed to establish differences between the English and simplified Chinese results.

\section{Results}

The simplified Chinese search websites were more likely to be commercial $(\mathrm{p}=0.045)$ and were more likely to not meet HONcode standards $(\mathrm{p}=0.008)$. No statistical significance was observed between the types of websites ( $\mathrm{p}=0.127)$, the prevalence of signs and symptom explanations $(\mathrm{p}=0.073)$, and the readability of the website $(\mathrm{p}=0.151)$.

\section{Conclusion}

The quality of websites obtained from the simplified Chinese character Google search was lower than those obtained from searches using the English language. Given the limited sources of language and culturally appropriate information on dementia, improvement of Internet resources may help to improve health outcomes of dementia patients in the Asian American population.

Received 10/19/2017 Review began 10/23/2017 Review ended 10/26/2017 Published 10/28/2017

(c) Copyright 2017

Tsiang et al. This is an open access article distributed under the terms of the Creative Commons Attribution License CC-BY 3.0., which permits unrestricted use, distribution, and reproduction in any medium, provided the original author and source are credited.
Categories: Medical Education, Neurology, Psychiatry

Keywords: chinese americans, asian americans, dementia, google, alzheimer's disease, health information, internet, prevention, chinese language, social media

\section{Introduction}

As the elderly population in the United States increases, the number of Americans diagnosed with dementia is likewise projected to grow. Data from the Alzheimer's Association estimates that at least one in ten adults age 65 and older is diagnosed with some form of dementia [1]. With Asian Americans as the fastest growing ethnic group over the last decade, it is becoming increasingly important for individuals in this ethnic community to be informed about this medical condition [2].

Although dementia cannot be completely cured, research shows that behavioral interventions such as cognitive rehabilitation or memory training are most effective in slowing the rate of cognitive decline when implemented in the early stages of the disease. Patients and family members who are more knowledgeable about dementia are more likely to recognize early symptoms and seek professional help during its onset. Unfortunately, studies have also revealed that Asian Americans are significantly less knowledgeable about dementia and are more likely to underutilize its treatment services [3-7]. Information about dementia accepted by this ethnic group often stem from unreliable sources such as personal observations, media, and oral tradition [8-10]. Thus, Asian Americans may be misguided in their beliefs about the symptoms and causes for dementia, which may hinder them from seeking professional help. This finding suggests that proper educational materials and services may be lacking among the Asian American community.

A recent study has found that although Chinese Americans hold misconceptions about dementia, their lack of knowledge does not reflect a lack of desire to become further educated about this medical condition [3]. Moreover, other recent studies have demonstrated that Chinese Americans are becoming more receptive to e-health and to obtaining dementia knowledge using the Internet than before [11-13]. In 2014, Google 
occupied $69 \%$ of the global search engine market and was the most popular search engine in the United States [14]. To the author's knowledge, there has been no research to date on the content and quality of dementia information available online in Chinese language. Our goal was to compare the content and quality differences between websites providing information on dementia as found by a Google search conducted both in simplified Chinese characters and in English.

\section{Materials And Methods}

A list of websites for this investigation was obtained by entering the term "dementia" into www.google.com/ncr for the English search. For the Google search on dementia conducted in simplified Chinese characters, the term “痴呆症” (chīdāizhèng), was entered into www.google.com/ncr, with simplified Chinese as the language used in search settings. The browsing history and cookies were cleared before each search, and the searches were conducted in Google Chrome's incognito mode to prevent browsers from remembering search activities. Each search was limited to the first three pages of results, as studies indicate that ninety-one percent of internet users do not go past the first page of search results [15]. The Google Chrome web browser was used to navigate each webpage. All videos and downloadable information were included in the evaluation. The information provided on links to external sites was not included in the evaluation.

The content of dementia websites was assessed in the following ways. First, the websites were divided into two categories according to the suffix and their affiliation statement: commercial or non-commercial. Second, each website was categorized into the following types of websites: informational, blog, news, and others (e.g., video, e-commerce, etc.) Third, the websites were reviewed for explaining signs and symptoms of dementia.

The quality of dementia websites was assessed for subjective readability and coherency in the respective search language. The Health On the Net Code of Conduct (HONcode) was also utilized to determine the quality of the websites. The HONcode is a process metric that evaluates medical and health websites in 35 languages with the following eight criteria: authoritative, complementarity, privacy policy, attribution (reference criteria and date), justifiability, transparency, financial disclosure, and advertising policy [16].

All data were entered and analyzed using IBM SPSS Statistics v. 21.0 (IBM, Armonk, NY). The results of the Google search conducted in simplified Chinese characters were compared with the results of the Google English search using chi-squared statistics to assess the content and to measure the quality of dementia information.

\section{Results}

The English google search of the term “dementia” generated 31 hits from the first three pages of search results. In terms of the content of dementia websites, one (3.2\%) of the websites was commercial. There were 18 (58.1\%) informational, four (12.9\%) blogs, four (12.9\%) news, and five (16.1\%) other types of websites. Twenty-one (67.7\%) websites explained signs and symptoms of dementia. For the English search, all 31 (100\%) websites were readable in the English language. Twelve (38.7\%) of these websites achieved the HONcode quality measures.

For the Google search conducted in simplified Chinese characters, there were also 31 results generated from the first three pages of search results. In terms of the content of dementia websites, six (19.4\%) of the websites were commercial. There were 12 (38.7\%) informational, five (16.1\%) blogs, seven (22.6\%) news, and seven (22.6\%) others types of websites. Fourteen (45.2\%) websites explained signs and symptoms of dementia. For the Chinese search, 29 (93.5\%) websites were readable in simplified Chinese characters. Three (9.7\%) websites achieved the HONcode quality measures.

In comparison with results from Google’s English search, the websites from Google's simplified Chinese search were more likely to be commercial (chi-square $=4.026, \mathrm{df}=1, \mathrm{p}=0.045$ ). No statistically significant difference was observed with the types of websites (chi-square $=2.325, \mathrm{df}=1, \mathrm{p}=0.127$ ) nor with whether the website explains the signs and symptoms of dementia (chi-squared $=3.215, \mathrm{df}=1, \mathrm{p}=0.073$ ). While the statistics did not reveal (chi-square $=2.067, \mathrm{df}=1, \mathrm{p}=0.151$ ) overall differences in readability, there were two websites in a different language found using the Chinese search, suggestive of poor search quality. There was a significant difference in the number of websites meeting the HONcode requirements between the two languages (chi-square $=7.123, \mathrm{df}=1, \mathrm{p}=0.008$ ).

\section{Discussion}

This study compared the content and quality differences between websites providing information on dementia as found by a Google search conducted both in simplified Chinese characters and in English. There were significant differences found between the results of English and simplified Chinese Google searches for the term "dementia". The results in simplified Chinese were significantly more likely to be commercial and were significantly less likely to meet HONcode quality requirements when compared to the results in English. 
The significant differences between the number of commercial websites appearing in simplified Chinese and English Google searches suggests that the search engine using simplified Chinese is less able to pinpoint relevant medical knowledge results for the user. This would likely cause more unrelated websites (commercial websites) to appear. For example, two of the six Chinese commercial websites in our search results were links to online marketplaces: one search result was for an anti-aging cream and other beauty products, and the other one was a wrist strap with the word "dementia" in Chinese. Additionally, two of the simplified Chinese Google search results were in a different language (not simplified Chinese). Thus, there is a need for further refinement of the Google search algorithms for simplified Chinese results.

For this study, we used HONcode qualifications as a proxy measurement for website quality. The significantly lower number of websites meeting HONcode qualifications suggests that websites for Chinese readers are less effective in providing appropriate and relevant health information. This lack of quality compounds the fact that Chinese readers, particularly those in the United States, already lack appropriate avenues for obtaining language and culturally appropriate health information on dementia $[3,5]$. It can thus be inferred that the lack of knowledge regarding dementia may also stem from a lack of adequate Internet sources, alongside many other issues. Future studies should assess stigma and attitudes toward dementia that are portrayed online in Chinese-language websites [17-20].

While there were no significant differences found between the number of websites explaining the signs and symptoms of dementia, only $45.2 \%$ of simplified Chinese websites did so, compared to $67.7 \%$ of English websites. This suggests that there is some room for improvement in the content of Chinese websites with regard to providing dementia information. As gender and age differences are associated with understanding the prognosis of dementia and identifying the causes of dementia, future studies should incorporate the effects of age and gender on Chinese-language website comprehension [21-22].

Due to the reliance on a search engine to produce the results, limitations of this study revolve around the deficits of the Google search algorithm. It is unknown how well optimized the search engine is for English and simplified Chinese results. The search engine algorithm ranks website relevancy non-randomly. Thus, the sample of websites on dementia obtained in this study is not representative of the entire population of available websites on dementia across the entire Web. The statistics provided should thus not be viewed as an embodiment of all websites on dementia, but rather as a measure of what users of the Internet are likely to see when performing a search on dementia in either language. Further studies should also utilize other search engines to replicate such results. As social media can bridge the gap between health care utilization and the Chinese over cultural barriers, future studies should examine the quality and content regarding dementia on social media [23-24].

\section{Conclusions}

Google search results for "dementia" in simplified Chinese, “痴呆症” (chīdāizhèng), are more likely to be commercial in nature and also of lower quality than Google results for "dementia" in English. Whether this is the result of a lack of optimization in the simplified Chinese search algorithm or a lack of quality websites for obtaining dementia information, the resulting void of information may contribute to the lack of knowledge on dementia and dementia treatment resources in Asian Americans. Our research suggests the need for the following further research: first, future studies should assess stigma and attitudes toward dementia that are portrayed online in Chinese-language websites; second, additional research should incorporate the effects of age and gender on Chinese-language website comprehension; lastly, further studies should examine the quality and content regarding dementia on social media. Ultimately, improvement of web resources on dementia can lead to improvements in knowledge and, therefore, outcomes of dementia in the Asian American community.

\section{Additional Information \\ Disclosures}

Human subjects: All authors have confirmed that this study did not involve human participants or tissue. Animal subjects: All authors have confirmed that this study did not involve animal subjects or tissue. Conflicts of interest: In compliance with the ICMJE uniform disclosure form, all authors declare the following: Payment/services info: All authors have declared that no financial support was received from any organization for the submitted work. Financial relationships: All authors have declared that they have no financial relationships at present or within the previous three years with any organizations that might have an interest in the submitted work. Other relationships: All authors have declared that there are no other relationships or activities that could appear to have influenced the submitted work.

\section{References}

1. 2017 Alzheimer's disease facts and figures . (2017). Accessed: October 4, 2017: http://www.alz.org/facts/ .

2. Census shows Asians are fastest-growing race group. (2012). Accessed: October 4, 2017: https://www.census.gov/newsroom/releases/archives/2010_census/cb12-cn22.html .

3. Ho EY, Woo BK: Dementia knowledge and information seeking of Chinese Americans . J Am Geriatr Soc. 2013, 61:647-8. 10.1111/jgs.12194 
4. Woo BK: Knowledge of dementia among Chinese American immigrants. Asian J Psychiatr. 2013, 6:351-2. 10.1016/j.ajp.2013.03.010

5. Diamond AG, Woo BK: Duration of residence and dementia literacy among Chinese Americans . Int J Soc Psychiatry. 2014, 60:406-9. 10.1177/0020764013491742

6. Nguyen PT, Nguyen N, Vo K, Ho S, Nguyen J, Woo BK: Knowledge of dementia among Vietnamese American immigrants. Asian J Psychiatr. 2016, 20:39-40. 10.1016/j.ajp.2015.11.005

7. Zheng X, Woo BK: Association between recognizing dementia as a mental illness and dementia knowledge among elderly Chinese Americans. World J Psychiatry. 2016, 6:233-8. 10.5498/wjp.v6.i2.233

8. Jones RS, Chow TW, Gatz M : Asian Americans and Alzheimer's disease: assimilation, culture, and beliefs . J Aging Stud. 2006, 20:11-25. 10.1016/j.jaging.2005.01.001

9. Woo BK: Using a Chinese radio station to disseminate dementia knowledge to Chinese Americans . J Am Geriatr Soc. 2012, 60:2175-6. 10.1111/j.1532-5415.2012.04230.x

10. Eng KJ, Woo BK: Knowledge of dementia community resources and stigma among Chinese American immigrants. Gen Hosp Psychiatry. 2015, 37:e3-4. 10.1016/j.genhosppsych.2014.11.003

11. Woo BK: Dementia health promotion for Chinese Americans. Cureus. 2017, 9:e1411. 10.7759/cureus.1411

12. Zheng X, Woo BK: E-mental health in ethnic minority: a comparison of YouTube and talk-based educational workshops in dementia. Asian J Psychiatr. 2017, 25:246-248. 10.1016/j.ajp.2016.12.002

13. Zheng X, Chung JO, Woo BK: Exploring the impact of a culturally tailored short film in modifying dementia stigma among Chinese Americans: a pilot study. Acad Psychiatry. 2016, 40:372-4. 10.1007/s40596-0150397-7

14. Google is easily the most popular search engine, but have you heard who's in second . (2014). Accessed: October 4, 2017: https://www.digitaltrends.com/web/google-baidu-are-the-worlds-most-popular-searchengines/.

15. van Deursen A, van Dijk J: Using the internet skill related problems in users' online behavior . Interact Comput. 2009, 21:393-402. 10.1016/j.intcom.2009.06.005

16. Boyer C, Appel RD, Ball MJ, et al.: Health on the net's 20 years of transparent and reliable health information. Stud Health Technol Inform. 2016, 228:700-4. 10.3233/978-1-61499-678-1-700

17. Woo BK, Mehta P: Examining the differences in the stigma of dementia and diabetes among Chinese Americans. Geriatr Gerontol Int. 2017, 17:760-764. 10.1111/ggi.12782

18. Dao NT, Woo BK: Gender differences in perceived stigma of dementia in Chinese Americans . Int J Geriatr Psychiatry. 2014, 29:987-8. 10.1002/gps.4125

19. Woo BK: Family history and its relationship with dementia stigma beliefs among Chinese Americans . Geriatr Gerontol Int. 2017, 17:122-125. 10.1111/ggi.12686

20. Woo BK, Chung JO: Public stigma associated with dementia in a Chinese-American immigrant population . J Am Geriatr Soc. 2013, 61:1832-3. 10.1111/jgs.12472

21. Siri M, Woo BK: Sex-related differences in understanding the prognosis of dementia . Geriatr Gerontol Int. 2014, 14:228-9. 10.1111/ggi.12125

22. Liu J, Woo BK: Older adults are less accurate than younger adults at identifying cardiovascular disease as a cause of dementia in the Chinese American community. Int Psychogeriatr. 2013, 25:1033-4. 10.1017/S1041610213000112

23. Woo BK: Comparison of mental health service utilization by Asian Americans and non-Hispanic Whites versus their cardiovascular care utilization. Cureus. 2017, 9:e1595. 10.7759/cureus.1595

24. Lam NH, Tsiang JT, Woo BK: Exploring the role of YouTube in disseminating psychoeducation . Acad Psychiatry. 2017, 1-4. 10.1007/s40596-017-0835-9 